\title{
Lesión renal aguda en pacientes con COVID-19 en la Unidad de Terapia Intensiva del Hospital Ángeles Pedregal
}

\author{
Acute kidney injury in patients with COVID-19 in the Intensive \\ Care Unit of the Hospital Ángeles Pedregal
}

\author{
Ivonne Martínez Martínez, * Humberto Ochoa Salmorán, \\ Adriana Enríquez Barajas, ${ }^{\S}$ Eduardo Teniza Frías, ${ }^{\S}$ Karla Vargas González, \\ Francisco Javier Padilla Pérez, "Enrique Juan Díaz Greene, II Juan Gerardo Esponda Prado** \\ Citar como: Martínez MI, Ochoa SH, Enríquez BA, Teniza FE, Vargas GK, Padilla PFJ et al. Lesión renal \\ aguda en pacientes con COVID-19 en la Unidad de Terapia Intensiva del Hospital Ángeles Pedregal. \\ Acta Med Grupo Angeles. 2021; 19 (2): 229-235. https://dx.doi.org/10.35366/100447
}

\section{Resumen}

En diciembre de 2019 se reportó en China la presencia de SARS-CoV-2, que la OMS denominó COVID-19, su manifestación principal es respiratoria. Se ha descrito que hasta $25 \%$ de los pacientes con SARS-CoV-2 desarrollan lesión renal aguda y la mortalidad de éstos es de $91.7 \%$. Objetivo: Describir las características de los pacientes infectados por COVID-19 que desarrollaron lesión renal. Material y métodos: Se estudiaron los pacientes admitidos en la Terapia Intensiva del Hospital Ángeles Pedregal que contaran con prueba positiva para SARSCoV-2. Se siguieron los valores de función renal de acuerdo con los valores de laboratorio de nuestra unidad. Resultados: Nuestro estudio consta de 22 casos, de los cuales 10 (45\%) no presentaron lesión renal aguda, 12 (55\%) lesión renal aguda, con la siguiente distribución: cuatro (18\%) AKI I, cinco (23\%) AKI II y tres (14\%) AKI III. Conclusiones: La incidencia de lesión renal aguda fue más alta que la reportada en la literatura, con $55 \%$ de los casos, requiriendo hemodiálisis 33\% de éstos. La mortalidad fue de $15.1 \%$ de acuerdo al SAPS-3, notablemente más baja que la reportada en la literatura.

Palabras clave: Lesión renal aguda, COVID-19, UTI.

\section{Abstract}

In December 2019 in China, the presence of SARS-CoV-2 was reported, which the WHO called COVID-19, its main manifestation is respiratory. It has been reported that up to $25 \%$ of patients with SARS-CoV-2 develop acute kidney injury and their mortality is $91.7 \%$. Objective: Describe the characteristics of patients infected by COVID-19 and who developed kidney damage. Material and methods: Patients admitted to the Intensive Care Unit at Hospital Ángeles Pedregal, who had a positive test for SARS-CoV-2, were studied. Renal function values were followed according to the laboratory values of our unit. Results: Our study consists of 22 cases, of which 10 (45\%) did not present acute kidney injury, 12 (55\%) with acute kidney injury, with the following distribution: four (18\%) AKI I, five $(23 \%)$ AKI II and three (14\%) AKI III. Conclusions: The incidence of AKI was higher than that reported in the literature, with $55 \%$ of the cases, requiring hemodialysis in $33 \%$ of the cases. Mortality was $15.1 \%$ according to SAPS-3, which was notably lower than that reported in the literature.

Keywords: Acute kidney injury, COVID-19, ITU.

\footnotetext{
* Médico Residente de Medicina Interna. Facultad Mexicana de Medicina, Universidad La Salle.

₹ Médico Intensivista adscrito al Servicio de Urgencias y Terapia Intensiva.

§ Médico Residente de Medicina Crítica. Facultad Mexicana de Medicina, Universidad La Salle.

^ Médico pasante del Servicio Social. Facultad Mexicana de Medicina, Universidad La Salle.

॥ Médico Internista, Profesor Titular de la Especialidad de Medicina Interna.

** Médico Intensivista, jefe de la Unidad de Terapia Intensiva.
}

Hospital Ángeles Pedregal. Ciudad de México, México.

Correspondencia:

Dr. Humberto Ochoa Salmorán

Correo electrónico: hochoas2528@gmail.com

Aceptado: 29-11-2020.

www.medigraphic.com/actamedica

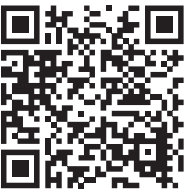




\section{INTRODUCCIÓN}

En diciembre de 2019 en la ciudad de Wuhan de la provincia Hubei, China se presentó una serie de casos de falla respiratoria aguda de origen desconocido que inicialmente se nombró síndrome respiratorio agudo severo por coronavirus 2 (SARS-CoV-2) por el agente etiológico que lo causa. En febrero de 2020 la Organización Mundial de la Salud (OMS) cambió oficialmente el nombre de la enfermedad causada por SARS-CoV-2 a enfermedad por coronavirus 2019 (COVID-19). La enfermedad rápidamente se extendió a zonas adyacentes a la ciudad de Wuhan y más tarde a otras ciudades y países. ${ }^{1,2}$

Reportándose el primer caso en la Ciudad de México el 29 de febrero de 2020. Para el día 22 de abril de 2020 en la Ciudad de México se contaban 64,431 casos confirmados, 13,882 casos sospechosos y 8,364 defunciones. $^{3}$

A pesar de que el daño pulmonar agudo es la principal manifestación de la enfermedad provocada por el COVID-19, se debe estudiar el daño a otros órganos. Una vez que se infecta el pulmón, el virus entra al torrente sanguíneo acumulándose en el riñón causando daño a las células renales. Se ha reportado que de 6.7 a $25 \%$ de los pacientes con SARS-CoV-2 detectado en sangre desarrollan lesión renal aguda (LRA) y la mortalidad se eleva hasta $91.7 \%$, este porcentaje es similar en pacientes con reacción en cadena de la polimerasa (RCP) para SARS-CoV-2 detectado en hisopado nasal. ${ }^{4,5} \mathrm{El}$ daño renal se puede ver en pacientes que desarrollan un síndrome de liberación de citoquinas demasiado intenso, además de signos de disfunción cardiaca con reducción de la fracción de eyección del ventrículo izquierdo. En ratones se demostró que el daño renal era secundario a apoptosis de las células del epitelio tubular renal. ${ }^{6,7}$ Los pacientes con COVID-19 que desarrollan falla orgánica son tratados esencialmente en las unidades de terapia intensiva.

El objetivo del presente trabajo fue determinar las características demográficas, clínicas y terapéuticas de los pacientes infectados por COVID-19 y que desarrollaron algún grado de lesión renal, así como su evolución en la Unidad de Terapia Intensiva (UTI) de nuestro hospital, resultados que se puedan extrapolar a otras unidades de terapia intensiva para lograr resultados similares.

\section{MATERIAL Y MÉTODOS}

Nuestro estudio cuenta con una muestra de pacientes admitidos en la Unidad de Terapia Intensiva del Hospital Ángeles Pedregal durante la pandemia de COVID-19 en la Ciudad de México en un periodo comprendido de abril a junio de 2020, los pacientes incluidos en el estudio debían contar con prueba positiva de reacción en cadena de polimerasa (RCP) para SARS-CoV-2, biometría hemática completa, dímero $D$, ferritina, proteína $C$ reactiva (PCR), velocidad de sedimentación globular (VSG), procalcitonina, química sanguínea y pruebas de funcionamiento hepático a su ingreso y con un seguimiento de los mismos durante su estancia en la Unidad de Terapia Intensiva. Se excluyeron expedientes incompletos o con laboratorios faltantes, también se excluyeron pacientes con antecedente de enfermedad renal crónica (ERC). En el caso de reingreso, se utilizaron los laboratorios de su primer ingreso como base para el estudio.

Se trata de un estudio longitudinal y descriptivo que tiene como objetivo determinar las características de los pacientes infectados por COVID-19 y que presentaron algún grado de LRA así como su evolución. La LRA se definió de acuerdo a las guías actuales con los siguientes datos: un aumento reciente de $1.5 \mathrm{mg} / \mathrm{dL}$ en el valor de la creatinina sérica, o $0.3 \mathrm{mg} / \mathrm{dL}$ o un decremento de $25 \%$ en la tasa de filtrado glomerular (TFG) estimada en los valores de base sostenidos por al menos tres días a pesar de una reanimación hídrica adecuada, además de un decremento de los volúmenes urinarios menor de $0.5 \mathrm{~mL} / \mathrm{kg} /$ hora por 6 horas. $^{8}$

Los valores de laboratorios tomados como normales fueron los señalados por el laboratorio de nuestra unidad.

Análisis estadístico. Describimos las características generales de nuestro estudio y también las particulares por grupo, entre quienes presentan lesión renal aguda y quienes no presentan lesión renal aguda. Hicimos un análisis descriptivo de la muestra observada usando frecuencias absolutas cuando las variables son cualitativas, acompañadas del porcentaje válido que representan según al grupo al que pertenecen. Cuando las variables son cuantitativas, usamos promedios y desviaciones estándar (DE) para describirlas.

\section{RESULTADOS}

Demográficos: nuestro estudio consta de 22 casos, de los cuales $10(45 \%)$ no presentaron lesión renal aguda (SLRA) y 12 (55\%) sí presentaron lesión renal aguda (CLRA). A partir de los 12 casos CLRA, encontramos la siguiente distribución de acuerdo con los grados: cuatro (18\%) CLRA AKI I, cinco (23\%) CLRA AKI II y tres (14\%) CLRA AKI III. En general, la muestra tuvo una estancia promedio de 18 días en la UTI. Al comparar los días de estancia por grupos, el grupo CLRA tuvo un promedio de casi 22 días considerablemente mayor que el grupo SLRA con un promedio de 14 días.

La edad promedio de pacientes ingresados a nuestro estudio fue de 60.7 años, sin existir un predominio de edad en los grupos CLRA y SLRA. El sexo masculino predominó en nuestro estudio con 77\% (17) pacientes ingresados, de los cuales $83 \%$ presentaron LRA. Analizando el IMC de 
los pacientes, observamos que dentro del grupo CLRA tres casos (25\%) tenían sobrepeso y cuatro (33\%) obesidad; en el grupo SLRA, dos casos (20\%) tenían sobrepeso y ocho (80\%) obesidad. Así, en general cinco (23\%) de casos del estudio ingresaron con sobrepeso y 12 (55\%) con obesidad.

\section{Comorbilidades}

Recabamos en la Tabla 1 información sobre enfermedades previas de los pacientes y su diagnóstico de ingreso a la UTI, encontrando algunos diagnósticos en el grupo CLRA como: un caso $(8 \%)$ con cardiopatía, un caso $(8 \%)$ con neoplasia y dos casos (17\%) con enfermedad pulmonar obstructiva crónica (EPOC). Para el grupo SLRA observamos: un caso $(10 \%)$ con administración previa de esteroides o inmunosupresores. Los diagnósticos previos más comunes fueron hipertensión arterial sistémica (HAS) y diabetes mellitus (DM) con nueve (41\%) y siete (32\%) de los casos, respectivamente.

Se observó que en la mayoría de los casos el diagnóstico principal fue insuficiencia respiratoria, difiriendo sólo en un caso (8\%) perteneciente al grupo CLRA con diagnóstico principal como estado de choque. Para el diagnóstico secundario, casi todos los casos fueron catalogados como neumonía por SARS-CoV-2, a excepción de un caso dentro del grupo SLRA (10\%), el ya mencionado estado de choque.

La carga viral estimada corresponde en su mayoría a leve y moderada, sin haber diferencia en los grupos CLRA y SLRA. Por último, se observó que el grupo CLRA presentó mayor sobreinfección con 10 (83\%) de los pacientes, contra tres $(30 \%)$ de los pacientes con sobreinfección del grupo SLRA.

\section{Características bioquímicas de la LRA}

En la Tabla 2 observamos que ocho (67\%) de los casos con lesión renal presentaron proteinuria contra cuatro pacientes $(40 \%)$ del grupo sin lesión renal aguda. Asimismo se siguieron a lo largo de su estancia en la UTI los valores de laboratorio de creatinina, urea y nitrógeno ureico en la sangre (BUN, por sus siglas en inglés), resultando notoriamente más elevados en los pacientes del grupo CLRA durante toda su estancia. Incluso al final con valores de casi dos veces más que el grupo SLRA. Esto va de la mano con los demás marcadores de inflamación.

En las tres gráficas de cajas y bigotes (Figuras 1 a 3) pudimos observar que la tasa de filtrado glomerular (TFG) fue notoriamente más alta para el grupo SLRA comparando un promedio de 91 contra un promedio de 43 del grupo CLRA; también podemos resaltar que la mínima que encontramos de TFG fue de 13. En la Figura 2 el índice neutrófilos/linfocitos (N/L) tiene un promedio de siete para el grupo SLRA contra un promedio de 10 para el grupo CLRA, aunque pareciera no ser tan grande la brecha de promedios, pudimos notar que la dispersión del índice en el grupo CLRA es muy amplia, tomando valores desde una mínima de uno hasta una máxima de 23, mientras que el grupo SLRA presentó un valor de índice N/L más concentrado. Por último, en la Figura 3, los promedios de la amplitud de distribución eritrocitaria (ADE) no se aprecian

Tabla 1: Enfermedades y diagnósticos previos.

\begin{tabular}{|c|c|c|c|}
\hline & Con lesión renal $(\mathrm{N}=12)$ & Sin lesión renal $(N=10)$ & Muestra total $(\mathrm{N}=22)$ \\
\hline & $\mathrm{n}(\%)$ & $\mathrm{n}(\%)$ & $\mathrm{n}(\%)$ \\
\hline \multicolumn{4}{|l|}{ Enfermedades previas } \\
\hline HAS & $6(50)$ & $3(30)$ & $9(41)$ \\
\hline DM & $4(33)$ & $3(30)$ & $7(32)$ \\
\hline \multicolumn{4}{|l|}{ Diagnóstico } \\
\hline Principal: insuficiencia respiratoria & $11(92)$ & $10(100)$ & $21(95)$ \\
\hline Secundaria: neumonía por SARS-CoV-2 & $12(100)$ & $9(90)$ & $21(95)$ \\
\hline Sepsis & $9(75)$ & $3(30)$ & $12(55)$ \\
\hline \multicolumn{4}{|l|}{ Carga viral estimada } \\
\hline Leve & $4(33)$ & $5(50)$ & $9(41)$ \\
\hline Moderada & $5(42)$ & $4(40)$ & $9(41)$ \\
\hline Severa & $3(25)$ & $1(10)$ & $4(18)$ \\
\hline Sobreinfección & $10(83)$ & $3(30)$ & $13(59)$ \\
\hline
\end{tabular}


Tabla 2: Características bioquímicas de la lesión renal aguda.

\begin{tabular}{|c|c|c|c|}
\hline & Con lesión renal ( $\mathrm{N}=12)$ & Sin lesión renal $(\mathrm{N}=10)$ & Muestra total $(\mathrm{N}=22)$ \\
\hline & & Media \pm desviación estándar & \\
\hline \multicolumn{4}{|l|}{ Creatinina } \\
\hline Inicial & $1.3 \pm 0.3$ & $0.8 \pm 0.2$ & $1.1 \pm 0.3$ \\
\hline Máxima & $2.1 \pm 1.3$ & $0.8 \pm 0.2$ & $1.6 \pm 1.1$ \\
\hline Final & $1.4 \pm 1.1$ & $0.6 \pm 0.1$ & $1 \pm 0.9$ \\
\hline \multicolumn{4}{|l|}{ Urea } \\
\hline Inicial & $49.5 \pm 26.3$ & $34.5 \pm 15.1$ & $43.1 \pm 23$ \\
\hline Máxima & $136.7 \pm 66.9$ & $70.4 \pm 36.2$ & $106.5 \pm 63.6$ \\
\hline Final & $68.6 \pm 63.8$ & $27.3 \pm 12.2$ & $49.8 \pm 51.3$ \\
\hline \multicolumn{4}{|l|}{ BUN } \\
\hline Inicial & $23.1 \pm 12.3$ & $17.2 \pm 7.5$ & $20.4 \pm 10.6$ \\
\hline Máxima & $63.9 \pm 31.2$ & $36.5 \pm 24.8$ & $51.4 \pm 31.1$ \\
\hline Final & $32.1 \pm 29.9$ & $12.7 \pm 5.7$ & $23.3 \pm 24$ \\
\hline Proteinuria, n (\%) & $8(67)$ & $4(40)$ & $12(55)$ \\
\hline
\end{tabular}

tan distantes, con un promedio de 12 para el grupo SLRA contra un promedio de 13 para el grupo CLRA, pero en la gráfica podemos observar que la distribución para el grupo CLRA es ligeramente mayor que los valores del otro grupo presentando un valor extremo máximo de 17.

\section{Tratamiento}

Para el tratamiento de la muestra en general se utilizaron: hidroxicloroquina y clexane en 20 casos (91\%), metilprednisolona en 18 casos (82\%) y tocilizumab en 16 casos (73\%) dentro de los más sobresalientes y sin diferencia entre los grupos SLRA y CLRA. De los pacientes CLRA, la hidroterapia fue el tratamiento más utilizado con 12 pacientes (100\%), el balance hídrico de estos pacientes fue de 12,875 mililitros, con un promedio de (13,818 mililitros), de los cuales cuatro $(33 \%)$ requirieron hemodiálisis.

No encontramos predominio de ninguno de los dos fenotipos de Gattinoni. Del total de casos sólo 17 (77\%) requirieron ventilación mecánica invasiva (VMI), ya divididos por subgrupos, la VMI se empleó en 11 (92\%) de los casos CLRA y sólo seis casos (60\%) en los pacientes SLRA. De igual forma, las maniobras de reclutamiento alveolar se llevaron a cabo en 10 (83\%) de los pacientes CLRA y sólo en cuatro $(40 \%)$ de los pacientes SLRA. En el grupo CLRA tuvimos cinco casos (42\%) que fue necesario el decúbito prono y dos (17\%) con óxido nítrico. Los pacientes CLRA también fueron aquéllos en los que se encontró en estado de choque, con uso de aminas en ocho (67\%) de los pacientes y de igual forma, con hipoxemia en ocho (67\%) de los casos. De los ocho casos que pertenecen al grupo
CLRA que observamos, fueron los mismos ocho casos con aminas y sólo seis permanecieron en la intersección de presentar también hipoxemia.

\section{Supervivencia y mortalidad}

De los cuatro pacientes que recibieron hemodiálisis, tres (25\%) estuvieron en modalidad intermitente y sólo uno (8\%) en modalidad de diálisis sostenida de baja eficiencia (SLED, por sus siglas en inglés). En el grupo CLRA, ocho casos $(67 \%)$ fueron dados de alta con vida contra nueve casos $(90 \%)$ del grupo SLRA, lo cual contrasta con una diferencia de 23 puntos porcentuales sobre el porcentaje de sobrevivencia en cada grupo. Las muertes han sido por falla orgánica múltiple. Sólo un caso (5\%) de la muestra total fue trasladado, este caso tenía LRA.

Por último, observamos que la mortalidad en el grupo CLRA fue de $15.1 \%$ ajustado con SAPS-3 y en el grupo SLRA fue de $7.1 \%$, en todos estos pacientes la causa de defunción fue falla orgánica múltiple.

\section{DISCUSIÓN}

La función renal está comprometida frecuentemente en los casos por infección por COVID-19, reportándose que más de $40 \%$ de los casos admitidos en hospitalización tiene algún grado de proteinuria. ${ }^{9}$ En nuestro estudio, 12 pacientes, $55 \%$ de la muestra, presentaron proteinuria detectada desde el ingreso. Siendo incluso más común en los pacientes que desarrollaron algún grado de LRA. De nuestra muestra, 12 pacientes, 55\% desarrollaron LRA. Se 
Figura 1: Tasa de filtrado glomerular.

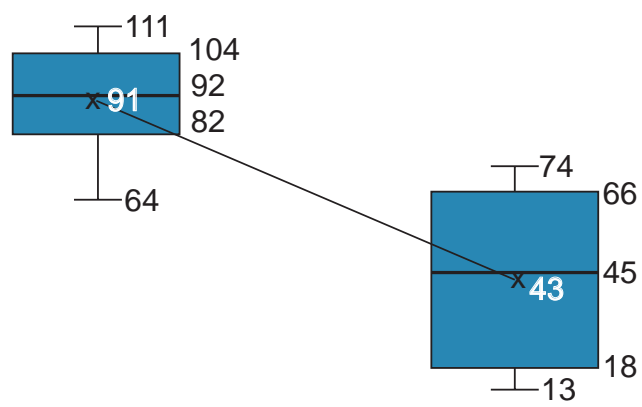

No

Sí

Lesión renal aguda

ha reportado que en la enfermedad grave, que requiere ingreso a la UTI, la LRA es relativamente común, afectando a $20-40 \%$ de los pacientes ingresados a esta área. Se considera que la presencia de LRA es un marcador de severidad de la enfermedad y un factor pronóstico negativo de sobrevida. ${ }^{9,10}$ De esta información se desprende la importancia de que se valore la función renal desde su ingreso, siendo ideal contar con valores de base de función renal de los pacientes en dicho momento.

La LRA en casi $20 \%$ de los pacientes requiere de tratamiento sustitutivo de la función renal (TSFR) en los pacientes admitidos en la UTI. ${ }^{11}$ En nuestra unidad, de los 12 pacientes que presentaron LRA, sólo cuatro (33\%) recibieron hemodiálisis, una cifra un poco mayor que la reportada en la literatura. La TSFR en nuestra UTI se dividió en las siguientes modalidades: tres (25\%) estuvieron en modalidad intermitente y sólo uno (8\%) en modalidad SLED. Si las estrategias conservadoras de tratamiento de la LRA fallan, se debe considerar el TSFR, especialmente en los pacientes con sobrecarga hídrica, en particular aquéllos con hipoxemia refractaria. En pacientes con LRA y COVID-19, el inicio temprano de TSFR y de soporte orgánico secuencial extracorpóreo garantiza un adecuado soporte orgánico además de que puede prevenir la progresión de la severidad de la enfermedad. La TSFR continua se prefiere sobre los modos convencionales por la inestabilidad hemodinámica de la mayoría de estos pacientes. ${ }^{12}$

Las maniobras de reclutamiento alveolar en pacientes sometidos a VMI se llevaron a cabo en 10 (83\%) de los pacientes CLRA y sólo en cuatro (40\%) de los pacientes SLRA. Está descrito el desarrollo de LRA en pacientes sometidos a ventilación mecánica, por lo que actualmente se definen estrategias de ventilación mecánica que puedan disminuir los efectos deletéreos de la VMI como el volutrauma y barotrauma, siendo la aplicación de las metas de protección pulmonar las medidas para disminuir los cambios hemodi- námicos y de citocinas a nivel renal. ${ }^{13}$ Es indudable el beneficio de emplear un volumen tidal (VT) bajo en pacientes con síndrome de insuficiencia respiratoria aguda (SIRA), el cual se considera ideal de $6 \mathrm{~mL} / \mathrm{kg}$ de peso, y se relaciona con un decremento de la mortalidad, disminución de días de internamiento, de días de ventilación mecánica invasiva y disminución de la incidencia de otras fallas orgánicas. ${ }^{14} \mathrm{Al}$ programar un VT bajo la aparición de LRA, en caso de que ocurra, lo hará de forma más tardía, esto por condicionar la elevación de los niveles de FNT- $\alpha$, IL-1 $\beta$, IL-6 e IL-8. EI beneficio de un VT bajo se explicaba por los niveles de hipercapnia moderada, la cual puede proteger a nivel renal, pero con los inconvenientes de las acidosis y la inestabilidad hemodinámica que deriva de la misma. ${ }^{15,16}$ Por otro lado, la presión final de espiración positiva (PEEP) elevada que en SIRA se ha demostrado es beneficiosa, desencadena alteraciones hemodinámicas con su respectiva repercusión orgánica. Annat y colaboradores han demostrado que con niveles de PEEP por arriba de $10 \mathrm{cmH}_{2} \mathrm{O}$ se presenta una disminución de volúmenes urinarios, flujo sanguíneo renal, excreción de sodio y potasio; estos cambios llegan a ser reversibles al disminuir la PEEP. ${ }^{15,17}$ La presencia de LRA se asocia a mayor tiempo de ventilación mecánica. Y a su vez los pacientes que recibieron VT más altos en los tres primeros días, tenían mayor riesgo de presentar LRA y de no recuperarse de ésta. ${ }^{18}$

El total de pacientes CLRA se trataron inicialmente con hidroterapia, siendo el balance hídrico final de 12,875 $(13,818)$. El tratamiento hídrico se recomienda se ajuste de acuerdo con la respuesta a volumen y a la tolerancia del mismo. El objetivo de esta recomendación es restaurar el volumen intravascular normal, evitando la sobrecarga de volumen, reduciendo la posibilidad de edema pulmonar, sobrecarga del ventrículo derecho, congestión y subsecuente LRA. La depleción de volumen es común al ingreso de estos pacientes por la presencia de fiebre, y el manejo

Figura 2: Índice neutrófilos/linfocitos.

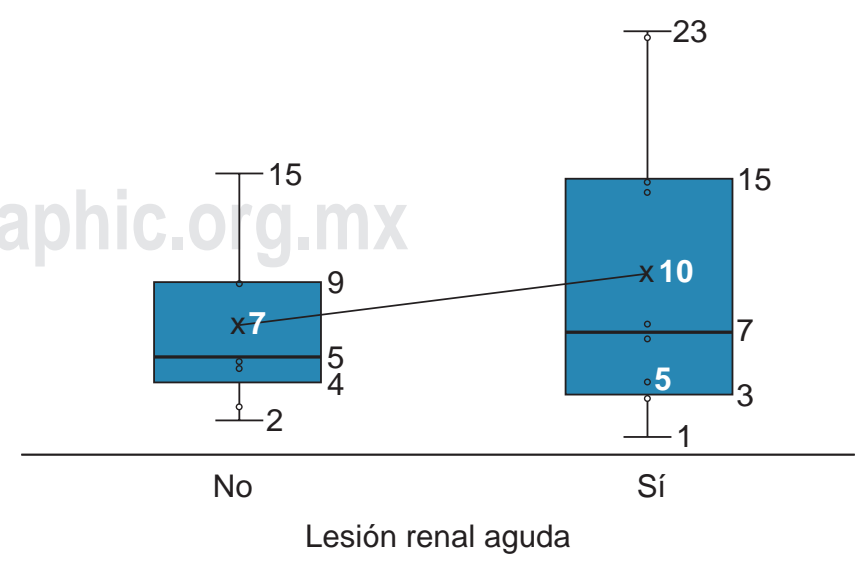


Figura 3: Amplitud de distribución eritrocitaria

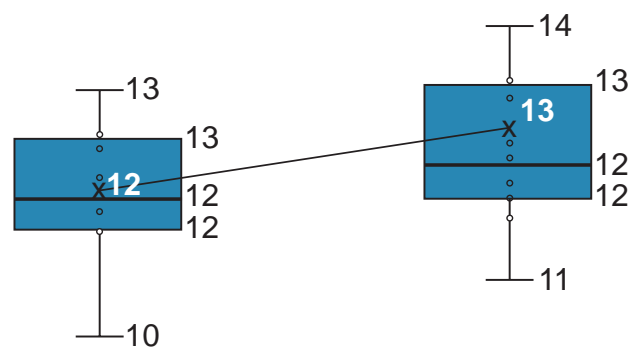

No

Sí

Lesión renal aguda

hídrico en el escenario prehospitalario rara vez se lleva a cabo. Por lo tanto, la terapia hídrica debe iniciarse de forma temprana para evitar la aparición de LRA. ${ }^{19}$

Además, en nuestro análisis encontramos que los pacientes CLRA presentaron un índice N/L ligeramente más alto, pero aún más importante mostraron una dispersión del índice N/L muy amplia con valores desde una mínima de uno hasta una máxima de 23. El índice N/L es un biomarcador fácilmente disponible de inflamación sistémica calculable a partir de una biometría hemática. Se puede interpretar como un reflejo del balance de la respuesta inmunitaria entre la respuesta innata (neutrófilos) y adaptativa (linfocitos). Los niveles elevados del índice N/L están asociados con el incremento de diversas citocinas proinflamatorias que pueden causar daño al ADN celular. ${ }^{20,21}$ Se ha demostrado el índice N/L como un predictor de mortalidad cardiovascular, supervivencia en enfermedad neoplásica, estado postoperatorio e incluso en progresión de la enfermedad renal crónica. En el caso de la LRA es innegable el papel de sustancias proinflamatorias en su fisiopatología, por tanto, el N/L deberá tener la capacidad de predecir el desarrollo de LRA. ${ }^{20}$

Por último, en relación con la ADE los pacientes en el grupo CLRA presentaron valores más elevados, Ilegando a ser en promedio de 17. El aumento de ADE refleja mayor heterogeneidad en el volumen de glóbulos rojos. Los trastornos relacionados con la producción ineficaz de coeficiente de variación de los glóbulos rojos circulantes (anemia por deficiencia de hierro, vitamina B12 y deficiencia de ácido fólico, supresión de la médula ósea y hemoglobinopatías) o aumento de la destrucción de éstos (hemólisis) o transfusión de sangre causarán una ADE más alto. ${ }^{22}$ Adicionalmente, el cambio de la ADE se ve afectado por muchos factores como disfunción hepática o renal, malnu- trición, cáncer, enfermedad tiroidea, respuesta inflamatoria aguda o crónica, uso de algunos medicamentos, activación del sistema renina-angiotensina y origen étnico. ${ }^{23-25}$ En el estudio de Kim y colaboradores se informó que los valores elevados de ADE son un marcador independiente de la mortalidad en los pacientes con sepsis o choque séptico. ${ }^{26}$

\section{CONCLUSIONES}

El desarrollo de LRA en pacientes con COVID-19 puede ser intrínseco a la patología de base así como al empleo de las estrategias terapéuticas. En este momento, cuando aún carecemos de un tratamiento específico, considerar todas las variables debe ser de importancia para iniciar estrategias preventivas como evitar sobrecarga hídrica, el empleo de VT o de PEEP elevados y cuando éstos se emplean para considerar sus efectos deletéreos; otra estrategia es la TSFR temprana. En la UTI del Hospital Ángeles Pedregal la incidencia de LRA fue más alta que la reportada en la literatura actualmente con 55\% de los casos, de éstos, $67 \%$ presentaron proteinuria, requiriendo hemodiálisis 33\% de los casos. La mortalidad del grupo CLRA fue de 15.1\% de acuerdo al SAPS-3, notablemente más baja que la reportada en la literatura.

\section{REFERENCIAS}

1. Zhu N, Zhang D, Wang W, Li X, Yang B, Song J et al. A Novel Coronavirus from patients with pneumonia in China, 2019. N Engl J Med. 2020; 382 (8): 727-733.

2. Lu R, Zhao X, Li J, Niu P, Yang B, Wu H et al. Genomic characterisation and epidemiology of 2019 novel coronavirus: implications for virus origins and receptor binding. Lancet. 2020; 395 (10224): 565-574.

3. https://covid19.cdmx.gob.mx/comunicacion/nota/reporte-21-dejulio

4. Huang C, Wang Y, Li X, Ren L, Zhao J, Hu Y et al. Clinical features of patients infected with 2019 novel coronavirus in Wuhan, China. Lancet. 2020; 395 (10223): 497-506.

5. Chu KH, Tsang WK, Tang CS, Lam MF, Lai FM, To KF et al. Acute renal impairment in coronavirus-associated severe acute respiratory syndrome. Kidney Int. 2005; 67 (2): 698-705.

6. Shimabukuro-Vornhagen A, Godel P, Subklewe M, Stemmler HJ, Schlober HA, Schlaak $M$ et al. Cytokine release syndrome. J Immunother Cancer. 2018; 6 (1): 56.

7. Tisoncik JR, Korth MJ, Simmons CP, Farrar J, Martin TR, Katze MG. Into the eye of the cytokine storm. Microbiol Mol Biol Rev. 2012; 76 (1): 16-32

8. Kellum JA, Lameire N; KDIGO AKI Guideline Work Group. Diagnosis, evaluation, and management of acute kidney injury: a KDIGO summary (Part 1). Crit Care. 2013; 17 (1): 204.

9. Cheng Y, Luo R, Wang K, Zhang M, Wang Z, Dong L et al. Kidney disease is associated with in-hospital death of patients with COVID-19. Kidney Int. 2020; 97 (5): 829-838.

10. Richardson S, Hirsch JS, Narasimhan M, Crawford JM, McGinn T, Davidson KW et al. Presenting characteristics, comorbidities, and outcomes among 5700 patients hospitalized with covid-19 in the New York City area. JAMA. 2020; 323 (20): 2052-2059. 
11. Zhou F, Yu T, Du R, Fan G, Liu Y, Liu Z et al. Clinical course and risk factors for mortality of adult inpatients with COVID-19 in Wuhan, China: a retrospective cohort study. Lancet. 2020; 395 (10229): 1054-1062. Erratum in: Lancet. 2020; 395 (10229):1038.

12. Husain-Syed F, Ricci Z, Brodie D, Vincent JL, Ranieri VM, Slutsky AS et al. Extracorporeal organ support (ECOS) in critical illness and acute kidney injury: from native to artificial organ crosstalk. Intensive Care Med. 2018; 44 (9): 1447-1459.

13. Ronco C, Bellomo R, Kellum JA. Acute kidney injury. Lancet. 2019; 394 (10212): 1949-1964.

14. Brower RG, Matthay MA, Morris A, Schoenfeld D, Thompson BT, Wheeler A. Ventilation with lower tidal volumes as compared with traditional tidal volumes for acute lung injury and the acute respiratory distress syndrome. N Engl J Med. 2000; 342 (18): 13011308.

15. Annat G, Viale JP, Bui Xuan B, Hadj Aissa O, Benzoni D, Vincent $M$ et al. Effect of PEEP ventilation on renal function, plasma renin, aldosterone, neurophysins and urinary $\mathrm{ADH}$, and prostaglandins. Anesthesiology. 1983; 58 (2): 136-141.

16. Andres-Hernando A, Dursun B, Altmann C, Ahuja C, He Z, Bhargava R et al. Cytokine production increases and cytokine clearance decreases in mice with bilateral nephrectomy. Nephrol Dial Transplant. 2012; 27 (12): 4339-4347.

17. Upadhyaya VD, Shariff MZ, Mathew RO, Hossain MA, Asif A, Vachharajani TJ. Management of acute kidney injury in the setting of acute respiratory distress syndrome: review focusing on ventilation and fluid management strategies. J Clin Med Res. 2020; 12 (1): 1-5.

18. McNicholas BA, Rezoagli E, Pham T, Madotto F, Guiard E, Fanelli V et al. Impact of early acute kidney injury on management and outcome in patients with acute respiratory distress syndrome: a secondary analysis of a multicenter observational study. Crit Care Med. 2019; 47 (9): 1216-1225.
19. Matthay MA, Aldrich JM, Gotts JE. Treatment for severe acute respiratory distress syndrome from COVID-19. Lancet Respir Med. 2020; 8 (5): 433-434.

20. Abu Alfeilat M, Slotkil I, Shavit L. Single emergency room measurement of neutrophil/lymphocyte ratio for early detection of acute kidney injury (AKI). Intern Emerg Med. 2018; 13 (5): 717-725.

21. Guthrie GJ, Charles KA, Roxburgh CS, Horgan PG, McMillan DC, Clarke SJ. The systemic inflammation-based neutrophil-lymphocyte ratio: experience in patients with cancer. Crit Rev Oncol Hematol. 2013; 88 (1): 218-230.

22. Bazick HS, Chang D, Mahadevappa K, Gibbons FK, Christopher KB. Red cell distribution width and all-cause mortality in critically ill patients. Crit Care Med. 2011; 39 (8): 1913-1921.

23. Hunziker S, Celi LA, Lee J, Howell MD. Red cell distribution width improves the simplified acute physiology score for risk prediction in unselected critically ill patients. Crit Care. 2012; 16 (3): R89.

24. Leekha S, Standiford HC. Empiric antimicrobial therapy for Gram-negative sepsis: back to the future. Crit Care Med. 2011; 39 (8): 1995-1996.

25. Balta S, Demirkol S, Akgul EO. Red blood cell distribution width is predictive of mortality in trauma patients. J Trauma Acute Care Surg. 2013; 75 (2): 345-346.

26. Ju XF, Wang F, Wang L, Wu X, Jiang TT, You DL et al. Dynamic change of red cell distribution width levels in prediction of hospital mortality in Chinese elderly patients with septic shock. Chin Med J (Engl). 2017; 130 (10): 1189-1195.

Conflicto de intereses: Los autores declaran no tener conflicto de intereses alguno.

Financiamiento: Los autores no recibieron patrocinio para llevar a cabo este artículo. 